\title{
Non-local turbulent transport: pollution dispersion aspects of coherent structure of convective flows
}

\author{
S. Zilitinkevich
}

Alfred Wegener Institute for Polar and Marine Research, Bremerhaven and Max Planck Institute for Meteorology, Hamburg, Germany

\begin{abstract}
During the last forty years vertical exchange in the atmospheric surface layer has been parameterized with the aid of the Monin-Obukhov similarity theory. Currently it is understood that the concept of local flux-gradient correspondence underlying that theory and most traditional turbulence closures breaks down in convective conditions. Physical essence of the problem is as follows. In strong convection large-scale semi-organised coherent structures embrace the entire convective boundary layer $(\sim 1 \mathrm{~km}$ in height). They generate pronounced largescale (2-3 km in the horizontal) flow patterns close to the surface, which play an important role in the horizontal dispersion of atmospheric pollutants. The largescale structures also yield local velocity shears and consequently the sheargenerated turbulence, which crucially affects heat/mass transfer. On the contrary, local shears, oriented in the opposite directions, only slightly affect the mean momentum transfer (hence the term "inactive turbulence"). Only first steps have been made in analysing these effects theoretically. In the present paper a review of the problem is given, and a new theoretical model is proposed capable of reproducing the above essential features of convection. The model is verified using data from recent large-eddy simulation of convective boundary layers together with the reference atmospheric data. Recommendations are given as to how to proceed in practical parameterization of the surface fluxes and liorizontal velocity variances in pollution dispersion models.
\end{abstract}

\section{Introduction}

Convective turbulence represents a very efficient mechanism of vertical transport of all kind of atmospheric pollution admixtures, as well as sensible 
heat and water vapour. In this paper we consider the transport properties of the convective surface layer that regulate vertical turbulent fluxes and horizontal dispersion of pollutants in the lowest several tens of meters of the atmosphere.

Traditional parameterizations of the surface-layer turbulence are based on the similarity theory of Monin and Obukhov ${ }^{1}$ and/or different level turbulence closures (e.g., those classified by Mellor and Yamada ${ }^{2}$ ). Theoretical concepts underlying these theories were formulated more than 20 years ago. Both the Monin-Obukhov theory and simple closures currently in use essentially employ the concept of a single-valued local correspondence between turbulent fluxes and mean gradients:

$$
\text { turbulent flux }=-(\text { eddy diffusivity }) \times(\text { mean gradient })
$$

which in turn implies the old-fashioned concept of turbulent flows as composed of fully organised mean component and fully chaotic component.

During the last decade it has been understood that the above concepts breaks down, first of all, in convective conditions. In place of simple distinction between fully organised and fully chaotic motions, an additional type of motion has become the concern of the theory, namely, large-scale coherent structures which are semi-organised (i.e. neither chaotic nor deterministic). These structures, being typical of convective planetary boundary layers (PBLs), play a key role in the PBL transport properties and make the nature of the PBL turbulence essentially non-local.

Physical nature of the non-local transport problem is especially clear in the shear-free convection regime. At very high Rayleigh numbers, coherent structures are the buoyancy-driven 3-dimensional Benard-type convective cells. They embrace the entire convective PBL $(\sim 1 \mathrm{~km}$ in height) and generate pronounced large-scale $(2-3 \mathrm{~km}$ in diameter) convergence flow patterns in the lower portion ( $<10 \mathrm{~m}$ in height) of the surface layer. This yields local velocity shears which crucially increase turbulent mixing close to the surface and, consequently, facilitate vertical exchange of scalar admixtures, heat, and water vapour. At the same time local shears are oriented in opposite directions and do not contribute directly to the mean-wind friction velocity. Accordingly, the effect of coherent structures on the mean momentum transfer is of secondary importance.

\section{Overview}

Traditional similarity theory for convection (Prandtl ${ }^{3}$, Obukhov ${ }^{4}$, Monin and Obukhov"), as well as the "1/3-power Nusselt/Rayleigh number heat/mass transfer law" widely used in engineering problems and occasionally used also in atmospheric problems (Malkus ${ }^{5}$, Golitsyn ${ }^{6}$, Siggia $^{7}$ ) are unable to reflect the effects of coherent structures. The point is that they employ one and the same 
local free convection velocity scale, $W_{c}$, for both vertical and horizontal fluctuations:

$$
W_{c}=\left(\beta Q_{s} z\right)^{1 / 3},
$$

where $z$ is the height over the surface, $Q_{s}$ is the potential temperature flux, $\beta=g / \theta_{0}$, is the buoyancy parameter, $g$ is the gravitational acceleration, and $\theta_{0}$ is the reference potential temperature. By contrast, horizontal velocity fluctuations associated with the PBL-scale near-surface convergence flow patterns should evidently be scaled by the Deardorff ${ }^{8}$ global convective velocity scale,

$$
w_{*}=\left(\beta Q_{s} h\right)^{1 / 3},
$$

where $h$ is the convective PBL depth.

Qualitatively the above features of convection were noticed already by Prandt $^{3}$ and subsequently by Kraichnan ${ }^{9}$ and Plate ${ }^{10}$.

More recently Businger ${ }^{11}$ emphasised vital importance of the nearsurface convergence flow patterns and pushed forward the concept of the friction velocity, $U_{*}$, generated by local horizontal velocity shears. $\mathrm{He}$ postulated that its area averaged value called "minimum friction velocity", $\left\langle U_{*}\right\rangle$, depends on the basic governing parameters of the convective PBL turbulence, $\beta, Q_{s}$, and $h$, and also on the roughness length of the underlying surface with respect to wind, $z_{0 u}$ :

$$
\left\langle U_{*}\right\rangle / w_{*}=\Phi_{*}\left(h / z_{0 u}\right)
$$

where $\Phi_{*}$ is a universal function expected to be monotonically decreasing with the increasing argument.

Basically the same physics underlies the old observation of Wyngaard and Cote ${ }^{12}$ and Panofsky et al. ${ }^{13}$ that horizontal velocity variances, $\sigma_{u}^{2}$ and $\sigma_{v}^{2}$, in an unstably stratified surface layer do not follow the traditional similarity theory. In the free convection limit they behave more or less as

$$
\sigma_{u}^{2}=\sigma_{v}^{2} \approx 0.5 w_{*}^{2},
$$

as distinct from vertical velocity variance that shows a very good agreement with the similarity theory prediction,

$$
\sigma_{w}^{2}=1.1 W_{c}^{2} .
$$

Here, the empirical estimate, 1.1, of the coefficient on the r.h.s. of eqn (5) is more or less trustworthy, confirmed both by field observations (Lenschow et al. ${ }^{14}$ ) and by numerical large-eddy simulation (LES) of convective PBLs 
(Moeng and Wyngaard ${ }^{15}$ ). The value of the coefficient on the r.h.s. of eqn (4) is much less reliable, ranging from 0.2 to 0.6 (see, e.g., Fig. 8 in Hibberd and Sawford ${ }^{16}$ ).

As already noted, large-scale structures, although contributing to $\sigma_{u}^{2}, \sigma_{v}^{2}$ and $\left\langle U_{*}\right\rangle$, scarcely affect the mean flux of momentum, which is why they can be refereed to as inactive turbulence (Townsend ${ }^{17}$, Bradshaw ${ }^{18}$, Högström ${ }^{19}$ ). Clearly, horizontal dispersion within the surface layer (through $\sigma_{u}^{2}$, and $\sigma_{v}^{2}$ ) and the heat/mass transfer at the surface (through $\left\langle U_{*}\right\rangle$ ) should essentially depend on such structures.

Pioneering quantitative models for the minimum friction velocity and related revision of the convective heat/mass transfer law have been developed by Schumann ${ }^{20}$ and Sykes et al. ${ }^{21}$, resulting in plausible expressions for the function $\Phi_{*}$, eqn (3), and the following formulations for the heat transfer law: Schumann,

$$
\Delta \theta w_{*} / Q_{s}=B_{1}\left(h / z_{0 u}\right)^{1 / 3},
$$

and Sykes et al.,

$$
\Delta \theta w_{*} / Q_{s}=B_{2} \ln ^{2}\left(\left\langle h_{s}\right\rangle / z_{0 u}\right)
$$

Here, $\Delta \theta$ is a conditional potential temperature increment across the convective layer (actually the difference between the so-called "surface aerodynamic temperature", $\theta_{0}$, and the air temperature far from the surface, $\left.\theta_{a}\right), Q_{s} / \Delta \theta w_{*}$ is the heat transfer coefficient, $z_{0 u} / h$ is the dimensionless roughness length of the surface with respect to wind, $\left\langle h_{s}\right\rangle$ is the area averaged depth of the near-surface horizontal convergence flow $\left(\left\langle h_{s}\right\rangle / z_{0 u}\right.$ being uniquely determined through $\left.z_{0 u} / h\right), B_{1}=0.6$ and $B_{2}=2.8$ are dimensionless constants calibrated by each author using his own LES. With the above constants, eqns (6) and (7) show good correspondence between each other and with the full range of LES data.

It turns out, however, that atmospheric data on the near-surface heat transfer reported by Stull $^{22}$ give

$$
\Delta \theta w_{*} / Q_{s}=160 \quad\left(z_{0 u}=0.005 m\right),
$$

which can reasonably be related to $z_{0 u} / h \approx 10^{-5}$. This dramatically diverges from the above LES data and model predictions.

Besides, no revision of the simple scaling formulation, eqn (4), for the horizontal velocity variance has been proposed to explain the great scatter of empirical data on $\left\{\sigma_{u}, \sigma_{u}\right\} / w_{*}$. In the present paper both issues, the heat/mass transfer and the horizontal velocity variance, are further investigated. 


\section{New model}

A plausible explanation for the above discrepancy between LES and atmospheric data lies in the fact that at present LES is unable to resolve the flow interaction with the surface roughness elements, and consequently, to distinguish between the roughness lengths with respect to wind, $z_{0 u}$, and to temperature, $z_{0 T}$. The Schumann ${ }^{20}$ and the Sykes et al. ${ }^{21}$ theoretical models do not distinguish between $z_{0 u}$, and $z_{0 T}$ as well.

To overcome the above impediments, a new model is proposed. It is based on the calculation of horizontal variability of the parameters in question, such as local depth, $h_{s}$, of the convergence flow pattern driven by a convective cell, local friction velocity, $U_{*}$, and the square of local horizontal velocity in a convective cell, $\bar{U}^{2}$. Than their mean values are determined as area averaged quantities, $\left\langle h_{s}\right\rangle,\left\langle U_{*}\right\rangle$ and $\sigma_{u}^{2}=\sigma_{v}^{2} \approx 0.5\left\langle\bar{U}^{2}\right\rangle$. Similarly, the heat and mass transfer laws are derived by area averaging of local relations between the fluxes and the real surface - air flow increments in temperature and scalar admixture respectively, taking the relevant roughness lengths, $z_{0 u}$, or $z_{0 T}$. The flow patterns are treated as internal boundary layers (IBLs) of basically radial geometry. Typical cell is taken axi-symmetric. Its properties are evaluated from simple scaling predictions (Sorbjan ${ }^{23}$, Zilitinkevich ${ }^{24}$ ) using the mass consistency requirement. Then the grows rate equation for the IBL height is solved together with local diagnostic resistance and heat/mass transfer laws borrowed from known self-similarity model of the IBL. Finally all local parameters are averaged in the horizontal plain over the entire area of convective cell.

In this way the following expressions are derived for the minimum friction velocity:

$$
<U_{*}>=\frac{A_{*} w_{*}}{\left[\ln \left(h / z_{0 u}\right)-B_{s}\right]^{1 / 3}},
$$

horizontal velocity variances:

$$
\sigma_{u}^{2}=\sigma_{v}^{2} \approx A_{U} w_{*}^{2}\left[\ln \left(h / z_{0 u}\right)-B_{s}\right]^{4 / 3},
$$

vertical flux of potential temperature:

$$
Q_{s}=\frac{A_{T}(\beta h)^{1 / 2}(\Delta \theta)^{3 / 2}}{\left[\ln \left(h / z_{0 u}\right)-B_{s}\right]^{1 / 2}\left[\ln \left(h / z_{0 T}\right)-B_{T}\right]^{3 / 2}},
$$

and vertical flux, $E_{s}$, of a scalar admixture characterised by its concentration, $q$ : 


$$
E_{s}=\frac{A_{q} \rho_{0}(\beta h)^{1 / 2}(\Delta q)^{3 / 2}}{\left[\ln \left(h / z_{0 u}\right)-B_{s}\right]^{1 / 2}\left[\ln \left(h / z_{0 q}\right)-B_{q}\right]^{3 / 2}} .
$$

Here, $\Delta \theta=\theta_{s}-\theta_{a}$ and $\Delta q=q_{s}-q_{a}$ are the real increments in potential temperature, $\theta$, and the scalar admixture concentration, $q$, between the underlying surface $\left(\theta_{s}, q_{s}\right)$ and the convective layer interior $\left(\theta_{a}, q_{a}\right) ; z_{0 u}, z_{0 T}$ and $z_{0 q} \approx z_{0 T}$ are the surface roughness lengths with respect to wind, temperature and the scalar in question, respectively; $\rho_{0}$ is the reference air density; $A_{*}, B_{s}, A_{U}, A_{T}, A_{q} \approx A_{T}, B_{T}$ and $B_{q} \approx B_{T}$ are dimensionless constants to be determined empirically

Eqn (9) for the minimum friction velocity agrees well with both the Schumann $^{20}$ and the Sykes et al. ${ }^{21}$ LES data (and also with their theoretical predictions) with the consequent estimates: $A_{*}=0.14$ and $B_{s}=5.7$.

In eqn (10) for horizontal velocity variance, the empirical constant $A_{U}$ was estimated as $A_{U}=0.25$ from the LES evidence on the shape of a typical convective cell, using reliable data on the intensity of vertical velocities within convective updraughts.

To determine the constants $A_{T}$ and $B_{T}$ in the heat transfer law, eqn (11), we are forced to use an indirect route. The point is that eqn (11) in its initial form can not be immediately verified using LES data, as LES actually confuse the genuine surface temperature, $\theta_{s}$, with the surface aerodynamic temperature, $\theta_{0}$, i.e. the air temperature extrapolated logarithmically downwards on the level $z=z_{0 u}$. Thus the reduced version of eqn (11) with $z_{0 u}$ substituted for $z_{0 T}$, namely

$$
\Delta \theta w_{*} / Q_{s}=A_{T}^{-2 / 3}\left[\ln \left(h / z_{0 u}\right)-B_{s}\right]^{1 / 3}\left[\ln \left(h / z_{0 u}\right)-B_{T}\right],
$$

was verified against the Schumann ${ }^{20}$ and Sykes et al. ${ }^{21}$ LES data to give $A_{T}=0.04$ and $B_{T} \approx B_{s}=5.7$.

For the surface roughness length with respect to temperature, $z_{0 T}$, a simple scaling model is developed resulting in the expression

$$
z_{0 T}=z_{0 u} \exp \left(-A_{0} \sqrt{\left\langle U_{*}\right\rangle z_{0 u} / v}\right),
$$

where $\left\langle U_{*}\right\rangle$ is the minimum friction velocity determined from eqn (9), $A_{0}$ is one more dimensionless constant. Then calibrating the complete heat transfer law, eqns (11) and (14), with the reference atmospheric value of the heat transfer coefficient, eqn (8), results in the estimate $A_{0}=0.8$. 


\section{Conclusions}

The main outcome of the proposed model is the heat/mass transfer law, eqns (11)/(12) with $z_{0 T}$ determined from eqns (14) and (9). Unlike the Schumann ${ }^{20}$ eqn (6) and the Sykes et al. ${ }^{21}$ eqn (7), it can be immediately used in atmospheric problems. In calm weather convection, it represents an alternative to both classical formulations, namely, the Monin-Obukhov similarity theory and the 1/3 power Nusselt/Rayleigh number law. Eqn. (10) for horizontal velocity variances can be used in calculation of horizontal dispersion in strongly unstable atmospheric surface layer.

\section{REFERENCES}

1. Monin, A.S. \& Obukhov, A.M. Basic laws of turbulent mixing in the atmospheric surface layer, Trudy Geofiz. Inst. Akad. Nauk SSSR, 1954, No. 24 (151), 163-187.

2. Mellor, G.L. \& Yamada, T. A hierarchy of turbulence closure models for planetary boundary layers, J. Atmos. Sci., 1974, 31, 1791-1806.

3. Prandtl, L. Meteorologische Anvendungen der Strömungslehre, Beitr. Phys. Fr. Atmos., 1932, 19, 188-202.

4. Obukhov, A.M. Turbulence in thermally inhomogeneous atmosphere Trudy Inst. Teoret. Geofiz. AN SSSR, 1, 95-115.

5. Malkus, W.V.R. The heat transport and spectrum of thermal turbulence, Proc. Roy. Soc. London, 1954, A225, 196-212.

6. Golitsyn, G.S. Simple theoretical and experimental study of convection with some geophysical applications and analogies, J. Fluid Mech., 1979, 95, 567-608.

7. Siggia, E.D. High Rayleigh number convection, Ann. Rev. Fluid Mech., 1994, 26, 137-168.

8. Deardorff, J.W. Convective velocity and temperature scales for the unstable planetary boundary layer, J. Atmos. Sci., 1970, 27, 1211-1213.

9. Kraichnan, R.H. Turbulent thermal convection at arbitrary Prandtl number, Phys. Fluids, 1962, 5, 1374-1389.

10. Plate, E.J. Aerodynamic Characteristics of Atmospheric Boundary Layer, U.S. Atomic Energy Commission, 1971.

11. Businger, J.A. A note on free convection, Boundary-Layer Meteorol., 1973, 4, 323-326.

12. Wyngaard, J.C. \& Cote, O.R. The evolution of a convective planetary boundary layer - a high-order-closure model study, Boundary-Layer Meteorol., 1974, 7, 289308.

13. Panofsky, H.A., Tennekes, H. \& Lenschow, D.H. The characteristics of turbulent velocity components in the surface layer under convective conditions, BoundaryLayer Meteorol., 1977, 11, 355-361.

14. Lenschow, D.H., Wyngaard, J.C. \& Pennell, W.T. Mean-field and second-moment budgets in a baroclinic, convective boundary layer, J. Atmos. Sci., 1980, 37, 13131326.

15. Moeng, C.-H., \& Wyngaard, J.C. Evaluation of turbulent transport and dissipation closures in second-order modelling, J. Atmos. Sci., 46, 2311-2330. 
16. Hibberd, M.F. \& Sawford, B.L. A saline laboratory model of the planetary convective boundary layer, Boundary-Layer Meteorol., 1994, 67, 229-250.

17. Townsend, A.A. Equilibrium layers and wall turbulence, J. Fluid Mech., 1961, 11, 97-120.

18 Bradshaw, P. Comments on "Horizontal velocity spectra in an unstable surface layer, J. Atmos. Sci., 1978, 35, 1768-1769.

19. Högström, U. Analysis of turbulence structure in the surface layer with a modified similarity formulation for near neutral conditions. J. Atmos. Sci., 1990, 47, 19491972.

20. Schumann, U. Minimum friction velocity and heat transfer in the rough surface layer of a convective boundary layer, Boundary-Layer Meteorol., 1988, 44, 311326.

21. Sykes, R.I., Henn, D.S. \& Lewellen, W.S. Surface-layer description under freeconvection conditions, Quart. J. Roy. Meteorol. Soc., 1993, 119, 409-421.

22. Stull, R.B. A convective transport theory for surface fluxes, J. Atmos. Sci., 1994, 51, 3-22.

23. Sorbjan, Z. Structure of the Atmospheric Boundary Layer, Prentice Hall, Englewood Cliffs NJ, 1989.

24. Zilitinkevich, S.S. A generalized scaling for convective shear flows, BoundaryLayer Meteorol., 1994, 70, 51-78. 